REVESCO. Revista de Estudios Cooperativos ISSN: $1885-8031$

\title{
MonedaPAR: una alternativa argentina para la economía social y solidaria
}

\author{
Eugenia Candelaria Pardo ${ }^{1}$
}

Recibido: 25 de marzo de 2019 / Aceptado: 30 de diciembre de 2019 / Publicado: 17 de junio de 2020

Resumen. MonedaPAR es una moneda digital argentina creada en 2017 complementaria a la moneda de curso legal. Al estar fundada sobre la tecnología blockchain se inserta dentro de los cambios tecnológicos globales, del debate sobre la especulación que roza a las criptomonedas y de la propia historia de monedas sociales en Argentina. Se desarrolla, a su vez, en un contexto recesivo de la economía con el anhelo de ser una alternativa para la economía social y solidaria, pequeñas y medianas empresas, empresas recuperadas y consumidores; toda vez que, aumentos en los precios de los alimentos, transporte, tarifas llevan a prescindir de bienes y servicios dejando a muchos productores sin compradores y viceversa. Con lo cual, verdaderamente pretende auspiciar las transacciones de bienes, servicios y saberes sobre la base de valores como la responsabilidad, la ayuda mutua y la solidaridad. El artículo propone, entonces, una lectura del contexto mundial, signado por una crisis financiera del capitalismo no resuelta, desigualdades sociales y cambios tecnológicos; pero también, se ofrece un panorama de las medidas económicas nacionales y su impacto en las organizaciones de la economía social y solidaria. Luego, se presenta la disyuntiva que suscitan las criptomonedas, junto a los objetivos, fortalezas, debilidades, amenazas y oportunidades que plantea MonedaPAR. Se hará, con una mirada descriptiva-interpretativa teniendo en cuenta investigaciones realizadas y en curso en el Instituto de Integración Latinoamericana -IIL- de la Universidad Nacional de La Plata -UNLP-; así como, entrevistas a referentes claves vinculados a la creación, desarrollo y divulgación de MonedaPAR. Permitirá reflexionar sobre las funciones de la moneda y las políticas e instrumentos necesarios para promover una mayor igualdad; de manera que, el mundo sea para todos y no para unos pocos, y la vida humana pueda dignificarse en su sentido más amplio.

Palabras clave: Crisis; Desigualdades; Tecnologías; Criptomonedas

Claves Econlit: E420; J54; P13.

\section{[en] MonedaPAR: an argentine alternative for the social and solidarity economy}

\begin{abstract}
MonedaPAR is an Argentine digital currency created in 2017 complementary to the legal tender. Being founded on the technology that provides blockchain is inserted within the global technological changes, the debate on the speculation that touches the cryptocurrencies and the history of social currencies in Argentina. It is developed in turn in a recessive context economy, with the desire to be an alternative for the social and solidarity economy, small and medium companies, recovered companies and consumers; whenever the increase in the prices of food, transport or rates leads to the dispensing of goods and services, producers will be left without buyers and vice versa. Thus, truly yearns to sponsor the transactions of goods, services and knowledge based on values such as responsibility, mutual aid and solidarity. The article proposes a reading of the global context, marked by an unsolved financial crisis of capitalism, social inequalities and technological changes; but also, it offers an overview of national economic measures and its impact on social and solidary economy organizations. Then is presented the dilemma raised by the cryptocurrencies, in addition to the objectives, strengths, weaknesses, threats and opportunities that MonedaPAR poses. It will be done with a descriptive-interpretative look considering researches carried out and in course at the Institute of Latin American Integration -IIL- of the National University of La Plata -UNLP; as well as interviews with key references linked to the creation, development and dissemination of MonedaPAR. It will make it possible to reflect on the functions of the currency and the policies and instruments necessary to promote greater equality; so that the world is for all and not for a few, and human life can be dignified in its broadest sense.
\end{abstract}

Keywords: Crisis; Inequalities; Technologies; Cryptocurrency.

Sumario. 1. Introducción. 2. Acerca del contexto: un panorama. 3. Criptomonedas: luces y sombras. 4. Reflexiones finales 5. Referencias bibliográficas.

Cómo citar. Pardo, E.C. (2020) MonedaPAR: una alternativa argentina para la economía social y solidaria REVESCO. Revista de Estudios Cooperativos, vol. 135, e69177. https://dx.doi.org/10.5209/reve.69177.

1 Facultad de Ciencias Jurídicas y Sociales. Universidad Nacional de la Plata, Argentina.

Dirección de correo electrónico: epardo@jursoc.unlp.edu.ar. 


\section{Introducción}

En un mundo con grandes desigualdades sociales, sumido en una compleja crisis del capitalismo no resuelta, y atrapado en una revolución tecnológica que pone en jaque al trabajo y a las sociedades; resulta urgente, una vez más, reflexionar qué tipo de políticas públicas o regulaciones son necesarias para salir de la crisis, cuáles pueden ser las nuevas formas de intervención pública para mitigar sus efectos, y qué tipo de alternativas pueden crearse para remediarla. Sin dudas, un escenario fértil para plantear las críticas al modelo de mundo en alza que no prioriza al ser humano ni el bienestar general; pero también, para pensar y ofrecer soluciones destinadas al fortalecimiento de las organizaciones de la economía social y solidaria toda vez que, promueven redes de solidaridad, valores y normas de beneficio colectivo, toma de decisiones democráticas, auspician la producción y distribución equitativa de bienes y servicios, la generación de empleo y sistemas de precios justos y de calidad.

Ello cobra aún más relevancia, cuando los gobiernos adoptan políticas de austeridad, de libre mercado, intensifican la concentración del ingreso, reprimarizan las exportaciones o condicionan la soberanía del Estado con nuevos ciclos de endeudamiento como actualmente sucede en Argentina. Sin dudas, el cambio de gobierno asestó un duro golpe contra trabajadores y productores al tiempo de implementar políticas económicas, monetarias y fiscales. Las medidas tuvieron como resultado una depreflación, depresión combinada con inflación, es decir, caída de la actividad económica sumado a inflación, deteriorándose los ingresos de los trabajadores, jubilados, pensionados, el empleo. Una combinación de políticas ortodoxas que se asociaron a las recomendaciones de austeridad y ajuste derivadas de la asistencia del Fondo Monetario Internacional -FMI- que asfixia, como al igual que todos los sectores, a la economía social y solidaria.

Sin embargo, aprovechando el desarrollo de la plataforma blockchain, en 2017 se puso en marcha la iniciativa MonedaPAR definida en su sitio web como una moneda digital complementaria a la moneda de curso legal. Por sus planteos y objetivos pretende ser un sistema alternativo para fomentar las transacciones de la economía social y solidaria; concediendo préstamos sin interés para adquirir bienes de consumo e insumos de producción; permitiendo satisfacer necesidades cuando aumentos en los precios de los alimentos, el transporte, o las tarifas llevan a prescindir de bienes y servicios dejando a muchos productores sin compradores y viceversa.

De manera que, el presente artículo propone una lectura del contexto mundial y nacional; luego, un panorama de la disyuntiva que suscitan las criptomonedas, junto a los objetivos, fortalezas, debilidades, amenazas y oportunidades de MonedaPAR para la economía social y solidaria. Se hará, con una mirada descriptiva-interpretativa teniendo en cuenta investigaciones realizadas y en curso en el Instituto de Integración Latinoamericana -IIL- de la Universidad Nacional de La Plata -UNLP-; así como, de voces de autores y de referentes claves vinculados a la creación, desarrollo y divulgación de MonedaPAR consultados a estos fines. En suma, permitirá reflexionar sobre las funciones de la moneda y qué políticas e instrumentos son necesarios promover y apoyar para una mayor igualdad, para que el mundo sea para todos y no para unos pocos.

\section{Acerca del contexto: un panorama}

Los acontecimientos financieros globales ocurridos en 2007-2008 visibilizaron las fisuras del régimen de acumulación sustentado en la valorización especulativa del capital; generador de profundas desigualdades y vulnerabilidades sociales. Sin dudas, puso en evidencia la ausencia de regulaciones nacionales y multilaterales junto a la necesidad de políticas para evitar su repetición (Schaposnik y Pardo, 2009, 2011 a y b). A tal punto que, en la reunión del G20 de abril de 2009, se reconoció que una crisis global exigía una solución global; pero, el compromiso sólo se limitó a "reparar" el sistema para restaurar el crédito, reforzar su regulación y aportar fondos a las instituciones financieras internacionales. Como sostiene Nahón (2018: 35) "el ímpetu transformador duró poco y finalmente primó una perspectiva más conservadora" obviando el nudo central del problema. Tampoco el FMI, pudo anticipar la crisis, sus plazos, su magnitud, ni advertir a sus miembros; con lo cual, era evidente el fracaso de las instituciones encargadas de velar por la estabilidad financiera y monetaria internacional.

En ese marco, en América Latina se reflexionaba sobre cuáles podían ser las nuevas formas de intervención pública; qué acciones podían llevarse adelante frente a la crisis; si era posible crear instituciones para remediarla; y, si las nuevas iniciativas regionales podían extender el perímetro de acción de los sectores públicos nacionales a modo de blindar a los países de sus efectos. Incluso, se consideraba que los gobiernos podían asumir un rol activo en el reparto de la riqueza a través de políticas públicas inclusivas en la 
integración regional, tal como lo aspiraba, por ejemplo, el Banco del Sur (Schaposnik y Pardo, 2009, 2011 a y b) ${ }^{2}$.

En lo esencial, resultaba esperanzador otro tipo de institución financiera porque las organizaciones de la economía social y solidaria no cuentan con financiamiento alternativo. Como sostiene Gambina (2013: 135) sabido es "que uno de los principales problemas de cualquier emprendimiento popular (pequeño o grande) pasa por el insuficiente acervo económico en capital de trabajo, que permita aplicar a la compra de materias primas, materiales e insumos para la actividad de producción y distribución" (...), de manera que "afectar fondos públicos para un banco de desarrollo supone reorientar financiamiento para el aliento de proyectos alternativos". Sin dudas, atender las necesidades de las cooperativas y organizaciones de la economía social y solidaria son necesarias; pero también, urgentes en una región caracterizada por profundas desigualdades sociales.

La cuestión es que, cuando transformaciones de este tipo no se producen; pero sí, cambian las condiciones políticas -como los de los últimos años en la región-, fiscales, o de comercio exterior, se inician nuevos ciclos de endeudamiento y los pueblos quedan en un estado de indefensión; al ser parte de políticas que pretenden condicionar, una vez más, la soberanía de los Estados (Pardo, 2018). Conforme con Rapoport (2018: 9), ellas hacen creer "en la existencia de un mundo integrado" (...) que busca, "eliminar cualquier obstáculo a los intercambios económicos y los flujos financieros por parte de los países más poderosos y las grandes corporaciones". Políticamente, supone el vaciamiento de la soberanía nacional, la violación de los procesos democráticos y la despolitización de las sociedades, lo que facilita la manipulación de sus gobiernos, utilizando distintos medios de desestabilización". Con lo cual, los sectores vinculados a la economía social y solidaria como cooperativas, pueblos originarios, campesinos, agricultores familiares, y otros actores sociales quedan invisibilizados ya que el modelo político, económico y social da facilidades y ventajas a empresas trasnacionales bajo la premisa de la competitividad.

Así que, los saberes y riquezas propias -otrora considerada por el colonialismo como inculta, ineficiente, primitiva, marginal- permanecen como 'ocultas' o 'invisibles' y por tanto marginadas. Se esgrime así, un modelo de mundo que refuerza la dependencia a un capitalismo cínico; cuyo cinismo para Sennet de Frutos (2012: 42) consiste en que "ya no pretende enmascararse las disfunciones del capitalismo para el cumplimiento del interés general apelando a recursos ideológicos"; sino que, se proclaman "las limitaciones estructurales del único sistema económico que se ha declarado posible, y que, dada la imposibilidad de las alternativas, no cabe sino aceptar y celebrar esa realidad limitada como buena y sin posibilidad de transformación".

De manera que, las desigualdades se manifiestan en la distribución desigual de poder y la desigual posibilidad de participar en el diseño de políticas públicas. Efectivamente, un puñado de "ciudadanos" locales y mundiales tiene el poder de cercenar los espacios de deliberación y de participación; generando aquello que García Linera (2015) denomina "procesos de ciudadanización neoliberales". Es también, lo que De Sousa Santos (Schijman, 2011: 4) llama "fascismo social", es decir, un régimen social que combina la democracia de muy baja intensidad con dictaduras plurales en las relaciones sociales, económicas y culturales", (...) donde "los grupos sociales dominantes adquieren un derecho de veto sobre la vida y las expectativas de grupos sociales oprimidos", (...) "no es un régimen político sino un régimen social y civilizatorio; promueve la democracia representativa al mismo tiempo que destruye las condiciones de ejercicio efectivo de los derechos democráticos de la gran mayoría".

Sin dudas, la crisis financiera de 2007-2008 no resuelta y extendida a lo económico logró contagiarse a todo el mundo reforzando estas tendencias. Con el agravante que, a diferencia de otras crisis del capitalismo, se trata para Ugarteche, Luna y Valencia (2016: 49), de una crisis de subconsumo, con rasgos conceptuales financieros que disparan elementos económicos; siendo el desplome, un subproducto del aumento de las tasas de interés de la Reserva Federal de Estados Unidos. Para los países de América Latina, ello significó la caída de los precios de los productos exportados; en tanto, para el mundo occidental la ralentización del crecimiento del consumo por no resolverse la crisis en términos productivos.

A la par, todo ello vino acompañado por una revolución digital -parte de un cambio tecnológico- que desafía a los gobiernos a pensar qué políticas podrían desarrollarse frente a las mejoras constantes en la productividad; pero también, sobre el futuro del trabajo. La CEPAL (2018: 15 y 16) advierte tres cuestiones importantes al respecto: por un lado, la expansión de las tecnologías digitales que desdibujan "los límites entre los sectores productivos de bienes y los de servicios", siendo los costos de los productos cada vez más difíciles de identificar y "utilizar en la medida en que el uso de los bienes físicos depende cada vez más de su conexión a 'la nube del producto"". Por otro, el retorno de la "discusión sobre los efectos de la economía de plataformas en la productividad"; y, por último, el desplazamiento de los trabajadores producto de las tecnologías aplicadas en el mundo del trabajo.

El Banco del Sur se propuso como parte de una nueva arquitectura financiera regional en América Latina en el seno de la crisis financiera internacional de 2007-2008. 
Dirksen (2019: 67) asevera que los cambios tecnológicos son importantes; pero que, el impacto de ellos en el mercado laboral, en la economía y sociedades como la latinoamericana es y será, una opción política. Efectivamente, en el mundo del trabajo "la precarización, y los intentos de desmontar los derechos laborales tanto a escala nacional como global empezaron mucho antes del auge de las plataformas o de la industria 4.0". Entiende que es ese proceso y no la tecnología la razón por la que la Organización Internacional del Trabajo -OIT- llama trabajo atípico a aquel precario, y trabajo autónomo o independiente a aquel mediante plataformas. La cuestión central por dirimir es, si los cambios tecnológicos funcionarán o no como un amplificador de las desigualdades sociales.

Junto a estas transformaciones, en Argentina comenzaron a producirse cambios en las políticas económicas que se intensificaron con el acuerdo con el FMI acordado en 2018 y condujeron a una caída de la actividad económica traccionada por la inflación, las tarifas y la devaluación que alcanzó el $300 \%$ desde inicios del 2016 a la fecha ${ }^{3}$. Efectivamente, pese al nulo incremento de la base monetaria en estricto cumplimiento de las metas acordadas, según el Informe elaborado por la Cooperativa de Estudios Scalabrini Ortiz -CESO- (2019: 4 y 5) la inflación mensual de octubre 2018 fue de 5,4\%, la de noviembre de 3,2\%, la de diciembre de $2,6 \%$ y la de enero 2019 de 2,9\%; en tanto, la interanual se ubicó en octubre $46 \%$, $49 \%$ en noviembre, $48 \%$ en diciembre y en enero acumuló el $50 \%$; con lo cual, las causas no obedecen a razones estrictamente monetarias. A ello se sumó el aumento generalizado y sostenido de las tarifas; en ese sentido, la infografía "Costo Tarifario", elaborada por la Universidad Nacional de Avellaneda -UNDAV- (2018) revela que desde inicios de 2016 se verificaron incrementos acumulados promedio del $2057 \%$ en gas natural, de $1491 \%$ en energía eléctrica y de casi un $1.000 \%$ en el servicio de agua potable ${ }^{4}$.

Justamente, estos aumentos provocaron una presión muy significativa en las pequeñas y medianas empresas y organizaciones de la economía social y solidaria. En ese sentido, Litvinoff (2018: párr. 2, 3 y 6) señala que los "tarifazos" asfixian al sector cooperativo y en particular, por ejemplo, "las empresas recuperadas, que hoy ya son más de 370 en todo el país, corren el riesgo de desaparecer; lo que representaría la pérdida de empleo para más de 25 mil personas, y un nuevo obstáculo en la lucha por generar espacios autogestivos que mantengan la dignidad y la igualdad en las condiciones laborales de trabajadores y trabajadoras"5. Efectivamente, cooperativas de la industria cerámica como Zanón, Cerámica Neuquén y Cerámica Stefani, Hotel Bauen, Cueroflex, Cristal San Justo, Textiles Pigüé, Cooperativa el progreso, Cooperativa Láctea Nuevo Amanecer, entre otras, están enfrentando serios problemas. A ella se suman, por ejemplo, la cooperativa Ininbox, una de las fábricas recuperadas más antiguas del país y la cooperativa de trabajo cristalería Vitrofin que están "cerradas hace ya varios meses porque no pueden ni siquiera prender los hornos debido al costo altísimo de la energía".

El antropólogo Ruggeri alerta que las actuales medidas económicas buscan "debilitar a la clase trabajadora, a bajar los salarios para beneficio de una economía destinada a la exportación de materias primas y de energía", además de estar al servicio de la especulación financiera. De manera que, al bajar el consumo, caer el empleo y el poder adquisitivo, baja la producción, baja la demanda, y "las cooperativas no pueden luchar contra eso, tienen que acomodarse. A eso hay que sumarle la apertura importadora" (Vaz, 2018: preg. 9). Entiende que la ofensiva, se relaciona con el alza de tarifas; pero también, con la apertura de las importaciones que "afectó la producción en casi todos los sectores, especialmente los industriales como textiles, cerámicos, metalúrgico, curtiembre, gráfica, cristal, etc. La baja del consumo, a prácticamente todos los demás. Esto tiene impacto directo sobre el ingreso y las condiciones de vida de los trabajadores y, por lo tanto, sobre la propia existencia de las recuperadas" (Ruggeri, 2018: párr. 3 y 4).

La CEPAL (2019: 84) registró en 2018 "un fuerte financiamiento del plan fiscal a través de la emisión de deuda, sumado a un tipo de cambio en continua depreciación"; lo cual, "hizo dudar a los inversores sobre la capacidad de la Argentina de hacer frente a los próximos vencimientos de la deuda", produciendo una corrida cambiaria y una fuga de capitales "tras la decisión de la Reserva Federal de los Estados Unidos de aumentar la tasa de interés". En este escenario negativo, el FMI aprobó un acuerdo stand-by -llevando la deuda pública nacional bruta al 77,4\%-, comprometiéndose Argentina a aplicar "un duro ajuste fiscal".

4 El informe de la UNDAV (2018: 3) añade que, en materia de transporte, se registraron "aumentos del $677 \%$ en peajes, $375 \%$ en el boleto del tren, $332 \%$ en colectivos de corta distancia y un $177 \%$ en subtes, para el acumulado trianual".

5 Se denominan empresas recuperadas a aquellas empresas capitalistas productoras de bienes y servicios que fueron declaradas en quiebra por sus dueños (incluso algunos "escaparon" y las abandonaron) dejando impagos varios meses de salarios y deudas. Fueron entonces, ocupadas por sus trabajadores reanudando parcialmente sus actividades bajo la forma cooperativas o asociaciones, gracias a una modificación a la ley de quiebras argentina.

6 Efectivamente, sabido es que la industria de la construcción tiene un efecto multiplicador en la economía y de acuerdo al Instituto Nacional de Estadísticas y Censos de Argentina -INDEC- $\left(2019^{\mathrm{a}}\right)$ el consumo de los insumos para la construcción en enero de 2019 muestran, con relación a igual mes del año anterior, bajas generalizadas en Pisos y Revestimientos cerámicos (-28.1\%), Artículos sanitarios de cerámica (-27,9\%), Ladrillos huecos $(-27,3 \%)$, Mosaicos graníticos y calcáreos $(-24,4 \%)$, Asfalto $(-22,8 \%)$, Cemento portland (-16,3\%), Cales y pinturas para construcción $(-15,7 \%)$, Hierro redondo y aceros para la construcción $(-15,2 \%)$, Placas de yeso $(-14,3 \%)$ y un $4,7 \%$ en el resto de los insumos (incluye grifería, tubos de acero sin costura y vidrio para construcción). Sólo, se registran subas de 5,7\% en yeso y 1,5\% en hormigón elaborado. Por su parte, el Índice de Producción Industrial manufacturero -IPI- de acuerdo con el INDEC (2019b), en el mes de enero de 2019 respecto a enero de 2018, evidencia una caída generalizada en todas las divisiones. Las mayores retracciones se dieron en Otro equipo de transporte ($58,8 \%)$, Maquinaria y equipo $(-42,8 \%)$, Productos textiles $(-27,9 \%)$ y Otros equipos, aparatos e instrumentos (- 26,3\%); le siguen luego, Productos minerales no metálico $(-19,0 \%)$, Vehículos automotores, carrocerías, remolques y autopartes (-18,0\%), Industrias metálicas básicas ($15,2 \%)$, Muebles y colchones, y otras industrias manufactureras $(-14,5 \%)$, Productos de caucho y plástico $(-12,4 \%)$, Prendas de vestir, cuero y 
Sabido es que, las organizaciones de la economía social y solidaria, las pequeñas y medianas empresas, son vulnerables y requieren políticas públicas de fomento porque contribuyen al desarrollo inclusivo, "mantienen los excedentes económicos en sus comunidades, contribuyen a la creación de empleos, prestan servicios allí donde la población lo requiere y promueven valores de solidaridad, integración social y compromiso democrático" (Schaposnik y Pardo, 2011 a: 302) ${ }^{7}$. Son estas características las que decidieron a la OIT y a la Organización de Naciones Unidas -ONU- a elevar recomendaciones a los gobiernos de todo el mundo para que apliquen políticas públicas de fomento hacia la economía social (OIT, 2001, 2002; ONU, 1998, 2001; Mendell, 2007; Depetris Guiguet y Rossini, 2008; Schaposnik, 2006, 2007).

\section{Criptomonedas: luces y sombras}

En estos escenarios, se insertó el diseño de una tecnológica como blockchain que ofrece seguridad e inviolabilidad en las transacciones. Se trata de un libro digital que descentralizada y públicamente contabiliza las transacciones. Sbatella (2017: 8) lo resume como "una contabilidad pública de persona a persona" que consta de tres partes fundamentales "una transacción, un registro de transacciones y un sistema que verifica y almacena la transacción". Como su nombre lo indica es una cadena de bloques que, explica Nadal (2017b: párr. 5), "permite identificar cada transacción encriptada y añadirla a la secuencia de los intercambios de tal forma que todos y cada uno de los nodos de la cadena actualiza su contabilidad instantáneamente" (...) "El registro histórico no puede ser alterado y entre más crece la cadena más se aleja la posibilidad de un ataque para perturbarla".

Ancladas en dicha tecnología, surgieron las monedas digitales en tanto medios de cambio electrónico que facilitan las transacciones. Entre sus atributos, conforme con Nadal (2017 a párr. 1 y 2) no estarían sujetas "al proceso de endeudamiento que caracteriza a la moneda emitida por los bancos comerciales privados al otorgar un crédito"; tampoco, al "respaldo por parte de un banco central". Dentro de ellas cobraron relevancia las criptomonedas, que ofrecen la posibilidad de emitirse y cambiarse de manos de forma descentralizada utilizando criptografía para mantener fidelidad, apoyándose en tecnologías de registro o libros contables (Barroilhet Díez, 2019) ${ }^{8}$. DE manera análoga, Preuskschat (2019, preg. 1) las define como "una forma de pago digital basada en la criptografía y cuyas transacciones, realizadas de forma anónima, se anotan en un libro contable distribuído". Justamente, ello le confiere sus "tres características esenciales: la seguridad, la descentralización y el anonimato".

Por su parte, Barroilhet Díez (2019: 30), advierte que este fenómeno es tan nuevo, que aún no se han desarrollado categorías conceptuales para regularlas. Con lo cual, surgieron otras expresiones paralelas al término criptomoneda empleada de manera indistinta tales como los tokens, criptotokens y criptoactivos. Por ejemplo, token hace referencia a una ficha y para Preuskschat (2017, preg. 2) "define a objetos similares a las monedas que dan derecho a disfrutar de una cantidad determinada de bienes y servicios, creados por el distribuidor de dichos bienes y servicios", siendo el caso de una ficha de una feria para subir a una atracción ${ }^{9}$. En el ámbito de las criptomonedas, se crearon los criptotokens que al igual que ellas según Navarro Cardoso (2019: 31) son "una unidad de valor aceptada por una comunidad y están sustentados en la tecnología blockchain" (...), no obstante, "los tokens tienen más funcionalidades". Tanto los criptotokens como las criptomonedas son criptoactivos.

Una de las criptomonedas más conocida es el bitcoin que, como medio de cambio y últimamente reserva de valor, planteó la necesidad de reflexionar sobre el rol del Estado en el comercio y las finanzas. Su aceptación generalizada en las transacciones en algunos países, posibilitó imaginarla como el instrumento de cambio del futuro; aunque, las fluctuaciones en las cotizaciones comenzaron a convertirla también como una reserva de valor y motivo de especulación. En ese sentido, Nadal (2017b) advierte sobre la vertiginosidad de los acontecimientos con la venta de bitcoin a futuro -y posiblemente próxima creación de derivados- a través de la empresa especializada Cboe Global Markets, uno de sus problemas más grandes, junto a la concentración de bitcoin en pocas manos -casi el $50 \%$ en circulación lo detentan mil personas-. Ello ha motivado a que distintas voces -como premios Nobel de economía-; e inclusive, gobiernos se inclinen por la regulación, o la aceptación como moneda de curso legal ${ }^{10}$.

calzado (-8,2\%), Sustancias y productos químicos (-7,4\%), Madera, papel, edición e impresión (-6,7\%), Refinación del petróleo, coque y combustible nuclear (- 4,9\%), \%, "Productos de metal" muestra una disminución de 4,1\%, Alimentos y bebidas (-2,2\%). Sólo registraron una suba los Productos de tabaco $(+5,1 \%)$.

Responde la cita a una traducción propia del inglés.

Dicho autor dentro de las monedas digitales distingue también otros similares como el dinero electrónico y las monedas virtuales.

Para Preuskschat (2017), las criptomonedas no son más que una modalidad de token.

10 Es el caso de China y Japón, según Massare (2017). En el caso de Japón, con un cambio en su legislación según Navarro Cardozo (2019:14) el bitcoin se convirtió un medio de pago, si bien las empresas que operan deben estar "registradas, cumplir con los requerimientos mínimos de capital, seguir las leyes contra el blanqueo de capitales y proteger la cuentas de cada uno de los usuarios por separado, para evitar robos masivos"; exigencias requeridas luego del robo de bitcoin a la casa de cambio Mt.Gox en 2013. 
Este tema, también estuvo presente en la Reunión de Ministros de Finanzas y Presidentes de Bancos Centrales del G20 realizada entre el 19 y 20 de mayo de 2018 en Buenos Aires en cuyo Comunicado se ratificó que los criptoactivos "carecen de los principales atributos que tienen las monedas soberanas"; planteando "cuestiones relacionadas con la protección de los consumidores e inversores, la integridad de los mercados, la evasión impositiva, el lavado de dinero, y el financiamiento al terrorismo" (pto. 9). En sentido similar, Bouveret y Haksar (2018: 27) entienden que "debido al carácter pseudoanónimo de la mayoría de las criptomonedas, son vulnerables a que se las emplee con fines de lavado de dinero y financiamiento del terrorismo, dado que ningún intermediario verifica la integridad de las transacciones o la identidad de quienes las efectúan".

Para Navarro Cardoso (2019: 17 y 29), la principal preocupación en cuanto al uso delictivo se centra en el uso como medio de pago del narcotráfico, en extorsiones y en el blanqueo de dinero, en cuyo caso el sujeto interesado en una criptomoneda ofrece dinero fiduciario a cambio de ella, pudiendo representar la colocación en una operación de lavado. Al respecto, los líderes del G20 reunidos en Japón en junio de 2019 se comprometieron a aplicar las recomendaciones formuladas por el Grupo de Acción Financiera -GAFIrelativas a la regulación de casas de cambio de criptomonedas, para prevenir el lavado de dinero y el financiamiento del terrorismo.

Valdecantos opina que, efectivamente, en el mundo de hoy las criptomonedas suelen "aparecer en los medios como una forma de acceder a enormes ganancias por medio de la especulación". Sostiene, además, que "algunos proyectos vinculados a criptomonedas han resultado ser estafas diseñadas para atraer inversores bajo la promesa de ganancias no realistas, a la vez que es factible que ciertos criptoactivos sean usados como mecanismo para facilitar actividades ilícitas, más allá de que no haya cifras sobre este aspecto". Sin embargo, a su entender "la importancia del fenómeno no pasa por la inversión especulativa en criptomonedas; sino, por la tecnología que se encuentra detrás: Blockchain" (...) que podría contribuir "a construir una sociedad más justa ya que mejoraría la economía de múltiples actores que hoy no tienen acceso a las formas tradicionales del dinero y el crédito. Estos objetivos sociales se encuentran en las antípodas de los aspectos planteados en el comunicado y en los medios masivos de comunicación"11.

Ciertamente, las criptomonedas pueden ser una alternativa para que, a nivel micro, los sectores excluidos de las políticas públicas puedan satisfacer sus necesidades vitales, e intercambiar bienes y servicios que de otra manera resultaría imposible; y, a nivel macro, podrían actuar como una fuerza anticíclica en momentos recesivos. Todo ello, propone también rediscutir, el para qué, y para quién del dinero fiduciario cuya confianza reposa en las autoridades monetarias, que ciertamente en este momento favorecen la actividad especulativa con reservas provenientes de endeudamiento y la colocación de títulos a mediano y largo plazo. Esto es lo que ha motivado a que, distintos autores y referentes de MonedaPAR como Mario Cafiero, propongan la necesidad de democratizar las políticas que toma la autoridad monetaria.

\subsection{Acerca de MonedaPAR}

En este contexto que imprimen los cambios globales tecnológicos se inserta MonedaPAR; pero a nivel nacional, lo hace dentro de la historia de las monedas no oficiales, alternativas que funcionaron hasta más de 14 años en Argentina y lograron rediscutir las funciones tradicionales de la moneda. Plasencia (2008: 16 y 17) reconoce una pluralidad de términos cercanos para aludir a las distintas experiencias que funcionan junto a las de curso legal. Por ejemplo, "monedas alternativas" suelen referirse a las monedas paralelas a la moneda oficial; "monedas comunitarias" son las creadas "por una comunidad preexistente, con adherentes formalmente constituidos, para su uso exclusivo dentro de dicho grupo"; "monedas complementarias" aluden a las "monedas locales o regionales, surgidas porque el sistema monetario legal es incompleto"; "moneda local", comprende a aquellas "vinculadas a territorios locales y regionales", que "surgen a partir de una asociación de personas (formal o informal) o bien por iniciativa gubernamental".

Si bien tienen en común ser diferentes a la moneda de curso legal, entre ellas existen fronteras borrosas. Plasencia (2014: 116) añade que se crean "para articular recursos subutilizados y disponibles con la satisfacción de necesidades que no se manifiestan en el mercado como demandas efectivas por falta de dinero oficial"; denominándose generalmente monedas complementarias -MC- "para subrayar que su intención no es sustituir a la moneda oficial sino llegar a donde esta no llega". A su vez, observa que para algunos autores un tipo de estas monedas, son las monedas sociales MSs "creadas para atender la inclusión de las personas que el mercado formal deja afuera"; en tanto para otros, las monedas sociales "son parte de un nuevo paradigma económico, porque tienen un potencial emancipatorio". Entiende que, las MSs dinamizan la economía popular y pueden impulsar la Economía social y solidaria -ESS-. Sin embargo, "otras MC que, sin ser MSs, pueden impulsar el desarrollo de la ESS" (...) y en ese sentido, observa que las

11 El Dr. en Economía Sebastián Valdecantos es co -fundador de MonedaPAR, co-CEO de WABA.network y actualmente economista jefe de MonedaPAR. Consultado en junio de 2018 a los fines de este artículo. 
criptomonedas son "una nueva generación de MC electrónicas", (...) "polémicas por servir a la ESS tanto como ser capaces de facilitar intercambios ilegales".

En particular, MonedaPAR se define como una moneda local destinada al comercio en una región limitada y complementaria a la de curso legal (el peso). Sbatella (2017: 7) entiende que "funciona complementando al dinero, en particular cuando la escasez de éste impide a muchas empresas, comercios y personas intercambiar sus capacidades" (...) "no es una moneda alternativa, sino complementaria al dinero de curso legal y al dinero bancario". Surgió en 2017 por iniciativa de cooperativas, pequeñas y medianas empresas, y empresas "recuperadas" con el objetivo de colocar sus productos en un contexto recesivo de la economía argentina.

Si bien, desde el punto de vista tecnológico para Valdecantos (2019: preg. 5), MonedaPAR es una criptomoneda, desde el punto de vista económico presenta diferencias respecto de Bitcoin o Ethereum, por ejemplo. En primer lugar, aclara que, "no tiene minado, por lo cual su funcionamiento no requiere del consumo de energía que requieren otras criptomonedas"; en segundo lugar, "funciona como una economía cerrada a los flujos financieros. Al no ser intercambiado en los Exchanges como el resto de las criptomonedas" (...), se puede "preservar la paridad 1 a 1 con el peso argentino, de manera tal de evitar las fluctuaciones que tienen la mayoría de las criptomonedas"; y en tercero, se apuesta a la tecnología "como un medio y no como un fin en sí mismo" 12 .

MonedaPAR se inspira en los principios elaborados por Silvio Gesell, quien como considera Valdecantos, (2018b: 2 y 3) "sugirió un tipo de moneda cuya circulación debería ser obligatoria o al menos fuertemente estimuladas como con la 'oxidación'"; otra forma sería a través de los sistemas de crédito mutuo. Propone discutir también, las funciones tradicionales asignadas al dinero, en especial la relativa a la función de reserva de valor que surge cuando se le asigna la función de unidad de cuenta y medio de cambio. Efectivamente, revela que esta forma de atesoramiento del dinero "es autodestructiva"; toda vez que, "no puede desempeñar exitosamente su función de medio de cambio": cuando hay certeza institucional de que mantendrá su valor a lo largo del tiempo, habrá incentivos para atesorarlo, sacarlo de circulación, y, por lo tanto, habrá menos transacciones. Además, esa función de reserva de valor implica que el dinero tiene un precio -la tasa de interés- y como puede cambiar, termina obstaculizando la función de unidad de cuenta.

Para MonedaPAR todos son "prosumidores" (productores-consumidores) que hacen un intercambio recíproco: cada miembro debe poner en el sistema (por la realización de ventas) al menos lo que saca del sistema (al hacer compras). Es coordinada por el ingeniero Mario Cafiero, del Observatorio de la Riqueza Padre Arrupe, organización que tiene por objetivo la puesta en práctica del mensaje del Papa Francisco plasmado en la Encíclica Laudato Sí y el Documento a los Movimientos Populares de Santa Cruz Bolivia. También, por Eduardo Murúa integrante del Movimiento Nacional de Empresas Recuperadas por los Trabajadores -MNER-, movimiento social que se encuadra en las luchas sociales por las consecuencias devastadoras de las políticas neoliberales aplicadas sistemáticamente durante los últimos 30 años del siglo XX: piqueteros, movimientos de desocupados, asambleas barriales; quienes bajo el lema "Ocupar, resistir, producir" lograron garantizar el proceso de recuperación de las empresas quebradas bajo la forma cooperativa. Por último, por el grupo técnico argentino Waba de desarrollo de criptomonedas.

En su sitio web se define como un sistema de intercambio de bienes, de servicios, de tiempo y también de crédito mutuo, que usa como medio cancelatorio de obligaciones a la moneda virtual MonedaPAR (\$PAR); e indica que funciona a través de la siguiente manera:

- Sistema de crédito mutuo: sus integrantes disponen de un monto de crédito, en forma de descubierto, sin interés, determinado por acuerdo de los miembros de la comunidad. Sbatella (2017: 7) añade que las empresas recuperadas tienen autorizado un crédito de 30.000 \$PAR, los productores individuales 10.000 \$PAR y los consumidores 1.000 \$PAR. Los miembros pueden avalar el ingreso de hasta 4 empresas y 20 consumidores; por lo que tiende a fortalecer la cadena de agregación de valor.

- Sistema basado en un intercambio reciproco: cada miembro dispone de una cuenta corriente individual. Si el saldo individual es positivo, ese miembro es acreedor del sistema, y puede gastar ese saldo en compras en el sistema. Si el saldo es negativo, ese miembro es deudor del sistema, y debe devolverlo vendiendo productos y servicios en el sistema. Añade Sbatella (2017) que un consumidor debe cancelar el saldo deficitario en un plazo máximo de 90 días; en tanto, productores y empresas disponen de 240 días.

Billetera digital: es el medio para realizar los pagos y cobros. Se trata de un sistema de cuenta basado en blockchain desde una aplicación móvil. La moneda utilizada en una transacción se crea en el momento de la transacción. La suma de todos los créditos sería como la base monetaria del sistema;

12 En su opinión, MonedaPAR “es un token pero también una criptomoneda". Considera que, "una criptomoneda es un caso específico de token"; es decir, "un subconjunto dentro del conjunto más amplio de los token", en tanto un "token" "es un activo programable digitalmente". Con lo cual, una criptomoneda "es un token cuyo caso de uso es específicamente monetario"; y en MonedaPAR, el PAR sería el token. (Consultado en noviembre de 2019 a los fines de este artículo). Podría entenderse entonces a MonedaPAR como un criptotoken. 
que se autorregula en función de los límites que fijan los mismos miembros que conforman el sistema. Las transacciones son entre personas o empresas; y no median intermediarios.

La administración del sistema PAR lleva la contabilidad de cada cuenta corriente y el sistema de clearing de todas las cuentas; cuyos saldos son de conocimiento público. Los precios, calidad, forma y plazo de entrega son de libre acuerdo entre comprador y vendedor. Al presente y dada su corta existencia, pueden resumirse las siguientes fortalezas, debilidades, oportunidades y amenazas de esta experiencia monetaria complementaria:

Cuadro. 1. Fortalezas-Debilidades-Oportunidades-Amenazas

\begin{tabular}{|l|l|}
\hline Fortalezas & Debilidades \\
-Confianza tecnológica. & -Cuestiones culturales. \\
-Transparencia. & -Desconocimiento. \\
-Practicidad y accesibilidad del sistema. & -Mercados acotados. \\
-Fortalece la cadena de valor. & -Poca difusión en medios hegemónicos. \\
-Crédito sin interés. & \\
-Aumenta el poder de compra. & \\
-Promueve el comercio local. & \\
-Fomenta el empleo. & \\
-Aumenta los clientes. & Amenazas \\
\hline $\begin{array}{l}\text { Oportunidades } \\
\text {-Necesidad de dinero para realizar compras. } \\
\text {-Política astringente del BCRA. }\end{array}$ & -Opiniones críticas sobre las criptomonedas en general. \\
-Nuevos actores en el mercado. & \\
-Nuevas capacidades. & \\
\hline
\end{tabular}

Fuente: Elaboración propia sobre la base de las entrevistas realizas e información disponible en: http://www.monedapar.com/.

La tecnología posibilita disponer información en tiempo real, inmutable, transparente y pública ya que se conoce en todo momento las tenencias de todos los participantes del sistema. La criptografía asegura la imposibilidad de falsificar la moneda y la billetera se descarga al celular funcionando como un banco "en el bolsillo". Valdecantos considera que "esta tecnología ofrece una serie de ventajas que van más allá del intercambio de valores digitalmente entre usuarios descentralizados. Blockchain tiene un enorme potencial para contribuir al bienestar de las comunidades" (...) "puede hacer superar las limitaciones que las monedas sociales suelen enfrentar, pudiendo generar sistemas confiables, transparentes, descentralizados y acordes al diseño organizativo de las comunidades que las gestionan"13.

Representa, además, una interesante política anticíclica generada e impulsada por intelectuales y movimientos sociales, que fueron más allá de la resignación o de la indignación, trabajando en el diseño de una alternativa. Justamente, observa Valdecantos que "la restricción de acceso al crédito que el sistema financiero impone a Pymes y pequeños productores, las condiciones de trabajo desfavorables para trabajadores informales y/o autónomos, y la capacidad de consumo no satisfecha en la población en general hacen que las iniciativas basadas en monedas complementarias como MonedaPAR cuenten con un potencial enorme para crecer y mejorar la economía de todos aquellos actores perjudicados por el estado actual de las cosas"14.

MonedaPAR se halla en una etapa de desarrollo, expansión y consolidación. Participan empresas recuperadas, como la Empresa Recuperada Industria Metalúrgica y Plástica Argentina Coop. Ltda. -IMPA-; asociaciones de pequeños productores como los Productores de Mar del Plata/Tandil etc.; la Asamblea de Pequeños y Medianos Empresarios -APYME-, y la Confederación Cooperativa de la República Argentina Cooperar-.

Si bien se desarrollaba en tres nodos: Gran Buenos Aires -GBA-, Mar del Plata y Tandil; comenzó a ampliarse funcionando actualmente en la Ciudad Autónoma de Buenos Aires, La Plata, Moreno, Lujan, Exaltación de la Cruz, Gualeguaychú, Capilla del Monte, Mendoza y Santa Ana. El nodo Mar del Plata, por ejemplo, surgió a partir de una charla a la cual asistió el Ingeniero Mario Cafiero, según relata Sladaga (2018) "con la finalidad de generar un encuentro de prosumidores para intercambiar bienes, servicios y saberes" (...). Ahí, "se conformó el nodo con varias organizaciones sociales como -APYME-; la Cooperativa

13 Consultado en 2018 a los fines de este artículo.

14 Consultado en 2018 a los fines de este artículo. 
Popular de Crédito Ltda. Nuevo Impulso; el Instituto Movilizador de Fondos Cooperativos -IMFC-; la Cooperativa de Trabajo Nuevo Amanecer; el proyecto social Proyecto Sierras - Espacio de Participación Ciudadana; y la Entramada" 15 . Una vez conformado el nodo, se discutieron formalidades y un reglamento que quedó plasmado en el denominado "Mercado de intercambio 2.0" ${ }^{16}$. Lo complejo, relata, es buscar asociaciones para intercambiar, por ejemplo, una cooperativa láctea que ingresa al sistema tiene que disponer de proveedores de insumos o materias primas. Para ello, es necesario que más organizaciones de la economía social y solidaria se vinculen e integren en cadenas de valor.

Una perspectiva de su evolución indica que a medida que fueron desarrollándose los distintos nodos las operaciones se incrementaron en cantidad y en pares sobre todo a partir del tercer trimestre de 2018, tal como muestra el cuadro a continuación; registrándose para el mes de octubre de 2019628 operaciones por un total de $292.726,48$ pares.

Cuadro.2. Operaciones (en cantidad)

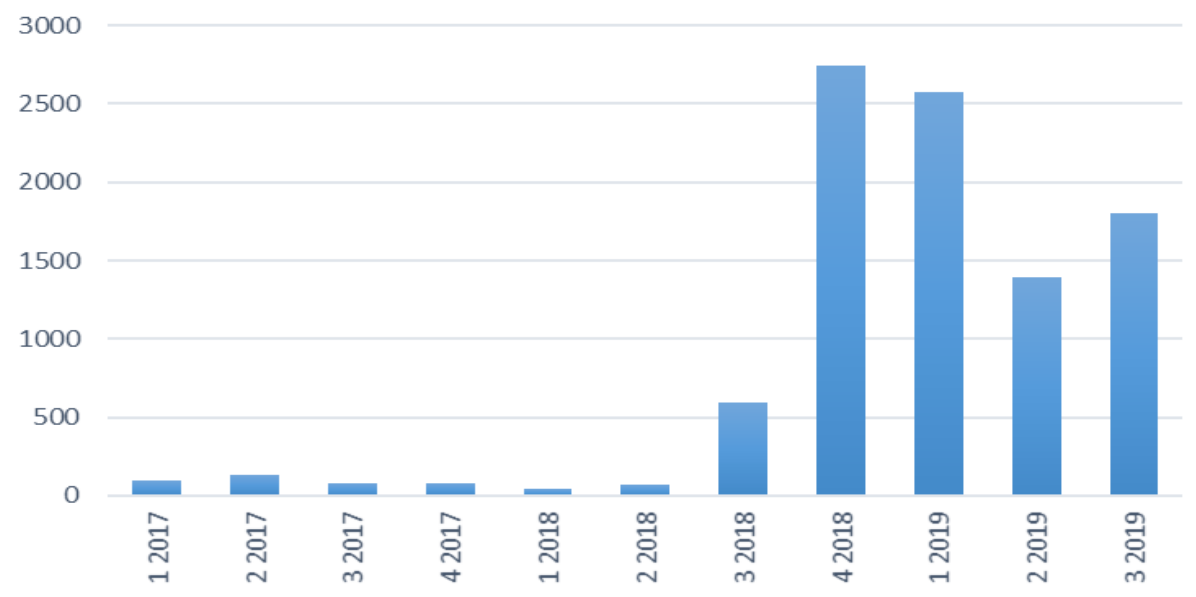

Fuente: Elaboración propia sobre la base de información extraída de blockchain proporcionada por MonedaPAR.

Cuadro.3. Operaciones (en pares)

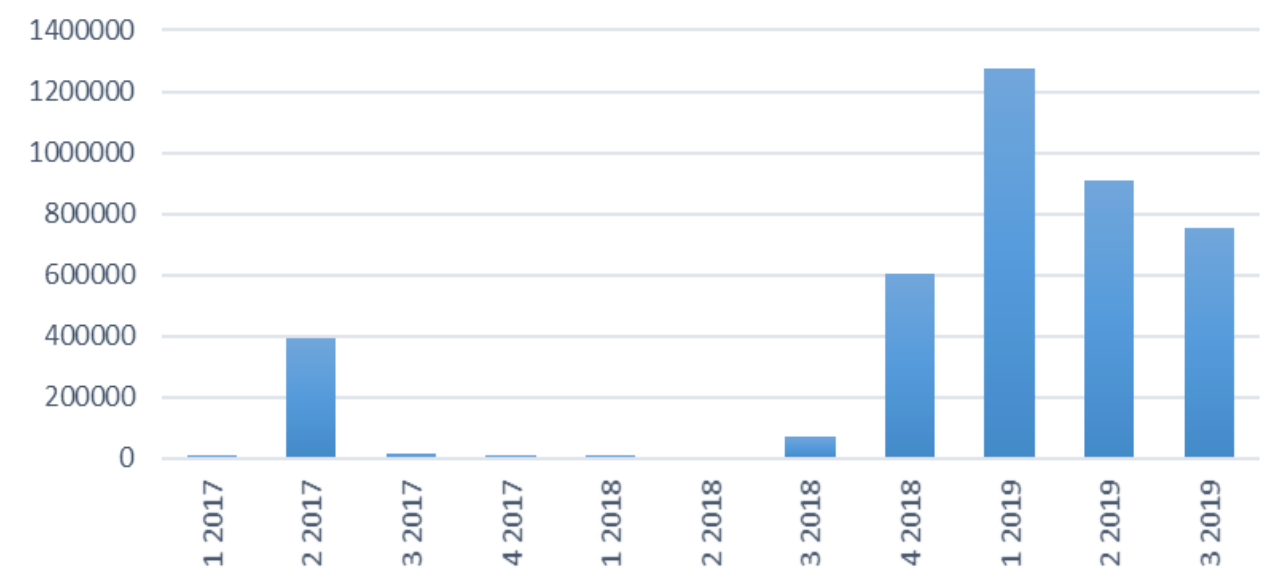

Fuente: Elaboración propia sobre la base de información extraída de blockchain proporcionada por MonedaPAR

15 La Entramada es una tienda colectiva que busca acercar diversos productos y sus productores a la sociedad local. Nace en el marco de Programa Entramados Productivos Latinoamericanos (EProL), Fundación EPyCA -Equipo de Producción y Construcción Alternativa- a fin de planificar necesidades, reservas, recursos y producciones populares, en función de la circulación organizada de los mismos. EProL desarrolla unidades productivas en forma entramada, mediante una unidad estratégica (plataforma productiva) móvil, en diferentes anclajes del país, donde EPyCA desarrolla el ensamblaje entre dos escalas (micro social / macro social), en procesos de producción, almacenamiento, agregado de valor, circulación, comercialización y consumo (https://www.facebook.com/pg/laentramadamdp/about/?ref=page internal).

16 Santiago Sladaga es militante social comprometido con la difusión y desarrollo de MonedaPAR, fue consultado el 29-6-2018 a los fines de este artículo. 
Más allá de la tendencia ascendente del uso de esta moneda digital interesa considerar aquellas debilidades que hicieron desaparecer a las monedas sociales en Argentina. Consultado Valdecantos opina que, es necesario superar las dificultades que se referían "con la falta de mecanismos y tecnologías confiables para garantizar una administración segura y transparente de las monedas". Las redes "necesitan, en general, un cierto grado de confianza entre los miembros que sólo se puede alcanzar en pequeñas comunidades". A medida que crece la red, el desafío es no poner en riesgo "la solidez de estos lazos". Por ejemplo, "los clubes de trueque que nacieron a mediados de los '90 y tuvieron su auge a comienzos de este siglo fueron perdiendo popularidad ante la circulación de cupones falsos y la emisión inadecuada de créditos oficiales", esta es sin duda, una de las cuestiones que deben mitigarse ${ }^{17}$. Por ello, es fundamental "dar a conocer tanto las ventajas de blockchain como las ventajas del crédito mutuo para las comunidades. Debido a que las formas de crédito y de dinero tradicionales están tan asentadas en nuestra forma de pensar, lograr que los potenciales participantes entiendan acabadamente el funcionamiento de los sistemas de crédito mutuo, basados en los principios de la economía social, es una tarea que requiere un esfuerzo activo por parte de todos los miembros de MonedaPAR"18.

De ahí que resulten vitales las tareas de difusión y divulgación. Por ello, se realizan charlas y presentaciones abiertas a la comunidad prosumidora y organizaciones sociales que se quieran incorporar a trabajar en una propuesta de intercambio con la criptomoneda. Comenta Valdecantos que los participantes de cada nodo realizan actividades de difusión dentro de sus comunidades para sumar nuevos participantes". Ello genera que "la iniciativa llegue a ser conocida por otros actores, que son contactados por WABA.network a fin de responder preguntas, realizar reuniones y eventualmente facilitar la incorporación de un nuevo nodo"19. Así, por ejemplo, se realizaron encuentros en Mar del Plata, que se difundieron en medios radiales, televisivos y virtuales: Twitter, Facebook y WhatsApp.

\section{Reflexiones finales}

Evidentemente MonedaPAR es una experiencia en desarrollo y expansión que, propone discutir las funciones del dinero y evidencia cómo un desarrollo tecnológico puede ofrecer soluciones para dinamizar el crédito, el consumo, el empleo fortaleciendo los lazos y valores cooperativos como los de solidaridad, la ayuda mutua, y la responsabilidad. Ciertamente anhela dar respuestas a necesidades de la economía social y solidaria, facilitando las transacciones de bienes, servicios y saberes, en un contexto recesivo de la economía argentina, donde el Estado no tiene voluntad de dialogar los problemas que aquejan a la sociedad, y no percibe las situaciones que están generando desajustes.

De manera que este tipo de experiencias pueden servir, como afirma Da Silva (2016), "como una especie de 'super yo', una especie de conciencia social de la sociedad para que la sociedad no se acomode a una situación de adaptación total donde ya no percibe los problemas que vive" (...) "se trata de una conciencia colectiva política de un conjunto de personas, de grupos "que se asocian de manera de decir ¡basta!, ¡no más de esta situación!". Sin dudas, una propuesta necesaria, urgente y posible, que puede contribuir a una mayor igualdad y a superar las vulnerabilidades sociales. Por eso el desafío para nuestros pueblos sigue siendo imaginar, apoyar y defender iniciativas como estas que pretenden la construcción colectiva de un mundo más igualitario, más justo y donde la vida se dignifique en su más amplio sentido.

\section{Referencias bibliográficas}

Barroilhet Díez, A. (2019) Criptomonedas, economía y derecho. Revista Chilena de Derecho y Tecnología, Vol. 8, № 1 , pp. 29-67. Disponible en: https://scielo.conicyt.cl/scielo.php?script=sci_arttext\&pid=S0719-25842019000100029.

Bouveret A. y Haksar, V. (2018) ¿Qué son las criptomonedas? La posibilidad de un nuevo tipo de dinero ofrece beneficios y comporta riesgos. Revista Finanzas y Desarrollo, junio 2018, pp. 26-35. Disponible en: https://www.imf.org/external/pubs/ft/fandd/spa/2018/06/pdf/fd0618s.pdf.

CEPAL (2018) La ineficiencia de la desigualdad. Síntesis. Disponible en: https://repositorio.cepal.org/bitstream/handle/11362/43566/4/S1800302 es.pdf.

CEPAL (2019) Balance Preliminar de las Economías de América Latina y el Caribe 2018. Disponible en: https://repositorio.cepal.org/bitstream/handle/11362/44326/141/S1801219_es.pdf.

17 Efectivamente, la experiencia de monedas sociales en Argentina había dejado enseñanzas que podían capitalizarse con esta plataforma tecnológica. Para Plasencia (2014), una de ellas fue "la dificultad en el manejo del circulante de MSs" (...) para ello, "resulta necesario conocer el número real de participantes; así como lograr que el circulante efectivamente circule y no se atesore, por ejemplo, a través de la 'oxidación'”. Otra, el control de la masividad, que "requería un tipo de gobernanza para la que nuestros sistemas no estaban diseñados. Nadie sabía en aquellos años de auge (nadie en el mundo) cómo gestionar MSs de uso masivo" (p. 119 y 120).

18 Consultado en 2018 a los fines de este artículo.

19 Consultado en 2018 a los fines de este artículo. 
CESO (2019) La economía argentina. Informe económico mensual. $\mathrm{N}^{\circ}$ II, febrero. Disponible en: https://www.ceso.com.ar/sites/www.ceso.com.ar/files/informe_economico_mensual_nro_ii_-_febrero_2019_completo.pdf.

COMUNICADO G20. Disponible en: http://www.bcra.gov.ar/Pdfs/Noticias/comunicado_G20_julio_2018.pdf.

Depetris Guiguet, E. y Rossini, G. (2008) Política cooperativa común en Mercosur. Algunas reflexiones sobre los alcances y dificultades", en Barrios D. (coord), Iniciativas asociativas y cooperativas en el Mercosur. Argentina: Edic. PROCOAS. pp. 17-34.

Dirksen, U. (2019) Trabajo del futuro y futuro del trabajo. Por una transición progresista. Revista Nueva Sociedad, $\mathrm{N}^{\mathrm{O}}$ 279, pp. 62-72. ISSN: 0251-3552. Disponible en: http://nuso.org/articulo/trabajo-del-futuro-y-futuro-del-trabajo/.

Gambina, J. (2013) Crisis del capital (2007/2013). La crisis capitalista contemporánea y el debate sobre las alternativas. Buenos Aires, Argentina: Edit. Fundación Investigaciones Sociales y Políticas - FISyP. 172 p.

García Linera, A. (2015) Ciudadanía y democracia en Bolivia, en Stefanoni P. (antología y presentación). La potencia plebeya: acción colectiva e identidades indígenas, obreras y populares en Bolivia. Buenos Aires, Argentina: Siglo XXI Edit. 173-196. http://biblioteca.clacso.edu.ar/clacso/coediciones/20100804092434/04cap3.pdf.

INDEC (2019a) Índice de producción industrial manufacturero. Vol. 3, $\mathrm{N}^{\circ} 36$. Disponible en: https://www.indec.gob.ar/uploads/informesdeprensa/isac_03_19.pdf.

INDEC (2019b) Indicadores de coyuntura de la actividad de la construcción. Vol. 3, $\mathrm{N}^{\circ} 37$. Disponible en: https://www.indec.gob.ar/uploads/informesdeprensa/ipi_manufacturero_03 19.pdf.

Litvinof, L. (2018) Cooperativas en riesgo de cierre por los tarifazos. Disponible en: http://www.revistacitrica.com/unveto-a-las-cooperativas.html.

Massare, B. (2017) La otra cara de la moneda. Entre el ideal libertario y la especulación financiera. Le Monde diplomatique, edición Cono Sur, $\mathrm{N}^{\circ}$ 221. Disponible en: http://www.eldiplo.org/221-neoliberal-y-popular/la-otracara-de-la-moneda/?token\&nID $=1$.

Mendell, M. (2007) Economía social y políticas públicas: el caso de Quebec, en: Vuotto M. (coord), La coconstrucción de políticas públicas en el campo de la economía social. Argentina: Editorial Prometeo. pp. 39-59.

Nadal, A. (2017a) Bitcoin y los tulipanes digitales. Diario la Jornada. Disponible en: http://www.jornada.com.mx/2017/08/23/opinion/023a1eco.

Nadal, A. (2017b) Bitcoin: entre mineros y ballenas. Diario la Jornada. Disponible en: http://www.jornada.com.mx/2017/12/13/opinion/022a1eco.

Nahón, C. (2018) América latina en el G-20: continuidades y rupturas de la agenda regional (2008-2018). Revista Voces en el Fénix, Año 8, N $\mathrm{N}^{\circ}$ 67. pp. 32-41. ISSN 1853-8819. Disponible en: http://www.vocesenelfenix.com/sites/default/files/numero_pdf/fenix67\%20baja_0.pdf.

Navarro Cardoso, F. (2019) Criptomonedas (en especial bitcóin) y blanqueo de dinero. Revista Electrónica de Ciencia Penal y Criminología. $\mathrm{N}^{\circ}$ 21, pp. 1-45. Disponible en: http://criminet.ugr.es/recpc/21/recpc21-14.pdf.

OIT (2001) Informe V: Promoción de las cooperativas. $89^{\circ}$ Conferencia, Ginebra, Suiza.

OIT (2002) Recomendación No 193 sobre la promoción de las cooperativas. $90^{\circ}$ Conferencia, Ginebra, Suiza.

ONU (1998) Papel de las cooperativas a la luz de las nuevas tendencias económicas y sociales. Informe $\mathrm{N}^{\circ}$ 54/57.

ONU (2001) Las cooperativas en el desarrollo social. Resolución A56/114 de la Asamblea General.

Pardo, E. C. (2018) Arquitectura financiera de América Latina. Más de 100 años de anhelos (1889-2009). Tesis de Maestría. Universidad Nacional de La Plata, Argentina. Disponible en: http://sedici.unlp.edu.ar/handle/10915/70391.

Plasencia, M. A. (2008) La oxidación monetaria y la moneda social. Aportes teóricos y análisis de un caso: la moneda social oxidable de Venado Tuerto, Pcia. de Santa Fe. Tesis de Maestría, Universidad Nacional de General Sarmiento. Disponible en: http://base.socioeco.org/docs/oxidacindelamoneda.plasencia.pdf.

Plasencia, M. A. (2014) Las experiencias de monedas sociales en la argentina. Revista Voces en el Fénix. $\mathrm{N}^{\circ} 38$. pp 114121. ISSN 1853-8819. Disponible en: http://www.vocesenelfenix.com/sites/default/files/numero_pdf/fenix67\%20baja_0.pdf.

Preukschat, A. (2017) Criptomonedas y tokens no son el mismo criptoactivo. Entrevista a José Antonio Bravo. Disponible en: https://www.eleconomista.es/economia/noticias/8724862/11/17/La-moda-de-crear-nuevos-bitcoinsdesde-Bitcoin-Cash-hasta-Bitcoin-Gold.html.

Rapoport, M. (2018) Sobre el libre comercio y las políticas de apertura del sector externo. Revista Voces en el Fénix. Año $8, \quad \mathrm{~N}^{\circ} \quad 67 . \quad$ pp. 6-15. ISSN 1853-8819. http://www.vocesenelfenix.com/sites/default/files/numero_pdf/fenix67\%20baja_0.pdf.

Romaguera, C. y Pou, A. (2015) ¡Que se vaya el patrón! 3 ejemplos de empresas recuperadas en Argentina. Disponible en: http://www.elsalmoncontracorriente.es/?Que-se-vaya-el-patron-3-ejemplos.

Ruggeri, A. (2018) Las empresas recuperadas después de dos años de nuevo neoliberalismo. Disponible en: http://autogestionrevista.com.ar/index.php/2018/01/31/las-empresas-recuperadas-despues-de-dos-anos-de-nuevoneoliberalismo.

Sbatella, J. (2017) La moneda virtual. Una experiencia reciente en Argentina. En IV Conferencia Internacional de Monedas Sociales y Complementarias. Dinero, conciencia y valores para el cambio social. Barcelona, España. Disponible en: https://drive.google.com/drive/folders/0B2v6wNA2wbaLdmhsY1ZjRW5BbWc.

Schaposnik, C. R. (2006) Las cooperativas, en: Mellado N. (coord.), Los actores empresariales argentinos frente al Mercosur. La Plata, Argentina: Ed. EDULP, La Plata. pp 75-93.

Schaposnik, C. R. (2007) Las cooperativas frente a las negociaciones del ALCA, en: Mellado N. (coord.), MERCOSURALCA. Articulación de las negociaciones internas y externas. La Plata, Argentina: Ed. Edulp. 
Schaposnik, C. R. y Pardo, E. C. (2009) Financiamiento y desarrollo en UNASUR, en: Mellado N (coord.), Mercosur y Comunidad Sudamericana de Naciones, ¿hacia dónde van? Córdoba, Argentina: Ed. Lerner. pp. 249-284.

Schaposnik, C. R. y Pardo, E. C. (2011a) Bank of the South, a Possible Alternative for Funding Souht American Development, en: Bance P. \& Bernier L. (comp.), Contemporary Crisis and Renewal of Public Action. Belgic: Edit PETER LANG SA. pp. 301-318.

Schaposnik, C. R. y Pardo, E. C. (2011b) Financiamiento a la economía social en la integración sudamericana. Banco del Sur y Banco del Alba: posibles aliados de las cooperativas. III Congreso Internacional de Investigación en Economía Social de CIRIEC: La Economía Social, pilar de un nuevo modelo de desarrollo-económico sostenible, CIRIEC Internacional-CIRIEC España-Universidad de Valladolid, ISBN 978-84-95003-85-0.

Schijman, B. (2011) Las izquierdas tienen que refundarse. Entrevista a Boaventura de Sousa Santos. Disponible en: https://rebelion.org/las-izquierdas-europeas-tienen-que-refundarse/.

Senent De Frutos, J. A. (2012) La dignidad humana: el horizonte utópico de los sistemas jurídicos inclusivos. Revista Éxodo, No 114. Disponible en: http://www.exodo.org/la-dignidad-humana-2/.

Soares Da Silva, A. (2016) Movimientos Sociales y Políticas Públicas. Clase 4, en Espacio de Formación Virtual, Diplomatura en Políticas Públicas para la igualdad en América Latina, Consejo Latinoamericano de Ciencias Sociales -CLACSO-. Disponible en: https://www.youtube.com/watch?v=ycVE1IDSOSA.

UNDAV (2018) Infografía costo tarifario. Disponible en: http://undav.edu.ar/general/recursos/adjuntos/22096.pdf.

Valdecantos, S. (2018b) MONEDA PAR: un sistema monetario al servicio de la producción y el trabajo. Disponible en: https://www.aciamericas.coop/IMG/pdf/2.3_sebastian_valdecantos.pdf.

Valdecantos, S. (2019). Es importante apostar a la organización de la sociedad, entrevista. Disponible en: https://www.galaxiay2k.com/tag/sebastian-valdecantos/.

Vaz, R. (2018) Realidades y desafíos de las empresas recuperadas en Argentina. Entrevista con Andrés Ruggeri, antropólogo e investigador. Disponible en: http://www.rebelion.org/noticias/2018/11/248977.pdf. 